\title{
Marcadores de alergia alimentaria en enfermedad péptica
}

\author{
EDUARDO TALESNIK G. ${ }^{1}$, DANIELA MAJERSON G. ${ }^{4}$, CAROLINA SERRANO H. ${ }^{1}$, \\ HELLY EINISMAN F. ${ }^{1}$, CARMEN GONZÁLEZ F. ${ }^{4}$, ALFREDO PEÑA V. ${ }^{1,3}$, \\ JAVIERA TORRES G. ${ }^{2}$, IGNACIO DUARTE D. ${ }^{2}$, PAUL R. HARRIS ${ }^{1}$ \\ 1. Departamento de Pediatría, Facultad de Medicina, Pontificia Universidad Católica de Chile. \\ 2. Departamento de Anatomía Patológica, Facultad de Medicina, Pontificia Universidad Católica de Chile. \\ 3. Hospital Dr. Sótero del Río. \\ 4. Internos de Medicina, Facultad de Medicina, Pontificia Universidad Católica de Chile.
}

\begin{abstract}
Food allergy markers in peptic disease

Background: The double-blind food challenge is the gold standard for diagnosis of food allergy, eventhough it is difficult to standardize and execute. An increase in allergy prevalence worldwide accentuates the importance of evaluating food allergy markers, in order to help the diagnosis. Objective: Elaboration of an operational definition for food hypersensitivity (FH) and evaluate the role of allergy markers, endoscopic and hystological findings, gastric mucosa cytokines and personal/family history of allergy in children. Method: Enrollment of children with suspected peptic disease referred for endoscopy. We obtained antral biopsies for hystological evaluation (eosinophil and mast cell count) and measurement of mucosal cytokines through an ELISA test. Patients were evaluated with Prick test, total serum IgE and clinical questionnaires for allergies. They were divided into two groups; children with and without food hypersensitivity. Results: 97 children were enrolled (mean: $11.7 \pm 3$, range 3-18). 4\% of children had FH. The endoscopic findings did not correlate with the presence of FH. $74.1 \%$ of patients without $\mathrm{FH}$ had eosinophils in the gastric mucosa compared to groups with FH which had $100 \%(p<0.05)$. Only IL-2 among the evaluated cytokines was found in a greater concentration in patients without $\mathrm{FH}$. $33 \%$ of patients considered themselves having history of personal allergies versus $11.8 \%$ of people without FH $(\mathrm{p}<0.05)$. Conclusions: $12,4 \%$ of children with digestive symptoms referred to endoscopy have FH. There are no clinical, endoscopic or hystological differences between patients with or without FH.

(Key words: allergy, hypersensitivity, food hypersensitivity, eosinophils).

Rev Chil Pediatr 2009; 80 (2): 121-128
\end{abstract}

Trabajo recibido el 20 de octubre de 2008, aceptado para publicación el 16 de febrero de 2009 .

Financiamiento: Proyecto Fondecyt \# 1030401

Correspondencia a:

Dr. Paul R. Harris D.

E-mail:pharris@med.puc.cl 


\section{RESUMEN}

Introducción: El diagnóstico de alergia a alimentos se fundamenta en la prueba de provocación oral doble ciego, de difícil estandarización y ejecución. El aumento de la prevalencia de alergia hace necesario la evaluación de marcadores de alergia a alimentos para facilitar el diagnóstico. Objetivo: Evaluar en niños, a partir de una definición operacional de hipersensibilidad a alimentos (HA), el rol de algunos marcadores de hipersensibilidad, hallazgos endoscópicos e histológicos, citoquinas de mucosa gástrica, y antecedentes personales y familiares de alergia. Métodos: Se enrolaron niños referidos a endoscopia por sospecha de enfermedad péptica. Se obtuvieron biopsias antrales para evaluación histológica (incluyendo eosinófilos y mastocitos) y citoquinas mediante ELISA. Se les realizó test cutáneo (TC), IgE total sérica y cuestionarios clínicos de alergia. Se dividió en 2 grupos, niños con y sin HA según criterio establecido. Resultados: Se reclutaron 97 niños (promedio: 11,7 \pm 3 años, rango 3 a 18). Un 12,4\% de los niños presentó HA. Los hallazgos endoscópicos no se relacionaron con la presencia de HA. Un 74,1\% de los pacientes sin HA presentó eosinófilos en la mucosa gástrica comparado con un $100 \%$ en el grupo con HA (p $<0,05)$. Sólo IL2 se encontró en mayor concentración en pacientes sin HA. Un 33,3\% de la población con HA consideró tener antecedentes personales de AA versus un $11,8 \%$ de los sin HA $(p<0,05)$. Conclusiones: La HA en niños referidos a endoscopia por síntomas digestivos está presente en un $12,4 \%$, sin elementos clínicos, endoscópicos o histológicos que los diferencien de niños sin HA.

(Palabras clave: alergia, hipersensibilidad, alergia alimentaria, eosinófilos).

Rev Chil Pediatr 2009; 80 (2): 121-128

\section{Introducción}

El aumento de la incidencia de alergias $^{1-3}$ ha sido atribuido a la menor estimulación del sistema inmune por antígenos microbianos que inducen una respuesta tipo T "helper" (Th1), produciendo un desbalance a favor de una respuesta $\mathrm{Th} 2$, la denominada teoría de la higiene. Factores involucrados incluyen la menor exposición a microorganismos, el menor número de hermanos, la limpieza del ambiente y el uso frecuente de antibióticos ${ }^{1}$. Se postula que las infecciones se asocian a mayor producción de Interleuquina (IL-12) e Interferón (IFN)- $\gamma$, que no sólo promueve un desbalance a favor de una repuesta tipo Th1, sino que también suprime la respuesta $\mathrm{Th} 2^{4,5}$.

La alergia a alimentos (AA) es una enfermedad caracterizada por una reacción de hipersensibilidad a antígenos de los alimentos, mediada por mecanismos inmunes ${ }^{6}$. Sus síntomas son variados e incluyen manifestaciones en la piel, el aparato respiratorio y gastrointestinal $^{7}$. El examen diagnóstico para AA es la prueba de provocación oral doble ciego. En cambio, la hipersensibilidad a alimentos (HA) es la presencia de marcadores que nos informan de sensibilización frente a un alimento, como ocurre con los test cutáneos, que a pesar de ser un método muy usado, sólo nos informan acerca de sensibilización IgE específica para el antígeno estudiado y no necesariamente se relaciona con síntomas ${ }^{8,9}$.

El aumento de prevalencia de alergia y su difícil estudio hace necesario la evaluación de marcadores de AA para así optimizar el diagnóstico. El objetivo de este trabajo fue evaluar en niños con síntomas digestivos sugerentes de organicidad clínica no asociados a AA, la frecuencia de algunos marcadores individuales de HA, su correlación con hallazgos endoscópicos e histológicos, con la concentración de citoquinas en la mucosa gástrica, y con antecedentes personales y familiares de alergia, utilizando como criterio diagnóstico una definición operacional de HA.

\section{Pacientes y Método}

Diseño del estudio. Entre el año 2002 y 2004 se enrolaron de manera prospectiva a niños menores de 18 años provenientes del Hospital Clínico de la Pontificia Universidad Católica de Chile (PUC) y del Hospital Sótero del Río, referidos a endoscopia digestiva alta 
por síntomas sugerentes de organicidad tales como hematemesis, pirosis/epigastralgia, dolor abdominal nocturno, vómitos crónicos asociados a la alimentación, sospecha de recidiva de úlcera péptica o dolor abdominal recurrente en un niño con un familiar directo con úlcera péptica. Se obtuvo el consentimiento informado de los padres para todos los pacientes. El estudio fue aprobado por el Comité de Ética de nuestras instituciones.

Endoscopia Digestiva Alta. El procedimiento fue realizado de manera estándar, describiendo la mucosa gástrica y duodenal como: normal, nodularidad antral, gastropatía, duodenopatía, úlcera gastroduodenal y otros. Se obtuvieron 3 biopsias gástricas y duodenales para el posterior estudio de citoquinas e histología. Una biopsia del antro fue utilizada para la detección de Helicobacter pylori mediante test de ureasa. Infección por la bacteria fue definida por la presencia directa a la visión histológica y/ o un test de ureasa positivo.

Histología. Los estudios histológicos se realizaron en el Departamento de Anatomía Patológica de la PUC. Las biopsias se fijaron en formalina al 10\%, se incluyeron en Paraplast y se tiñeron con hematoxilina eosina. La estimación de infiltración por eosinófilos se evaluó mediante 2 sistemas: uno semi-cuantitativo y otro cuantitativo. En el primero se dividió la infiltración en 4 grados según presencia de eosinófilos en la lámina propia. En el segundo se contaron los eosinófilos, calculando la media por milímetro cuadrado de mucosa gástrica. Para el estudio de mastocitos en cortes desparafinizados se procedió al desenmascaramiento de antígenos con buffer EDTA a $\mathrm{pH} 8,0$, en horno microondas. Se practicó reacción inmunohistoquímica con el anticuerpo anti-mast cell tryptase, clon AA1 (Novocastra), con el sistema de revelado automático I-6000 (Biogenex) que utiliza streptoavidina-biotina y diaminobenzidina (DAB) como sustrato cromógeno. Se contaron las células que presentaban caracteres morfológicos de mastocitos y gránulos citoplasmáticos positivos para triptasa, y se calculó su media por milímetro cuadrado ${ }^{10}$.

Citoquinas. Para la determinación de citoquinas cada biopsia antral fue homogenizada con un disruptor de tejidos (OMNI Th inter- national) separadamente en 750 ul de suero fisiológico. Los sobrenadantes obtenidos en una centrifuga mini Eppendorf (12 $000 \mathrm{~g}$ por $5 \mathrm{mi}$ nutos a $4{ }^{\circ} \mathrm{C}$ ) fueron congelados a $-70{ }^{\circ} \mathrm{C}$ en criotubos estériles (Sardstest) hasta su utilización en los ELISA. Se determinó el contenido proteico total usando una modificación del método del ácido bicinconinico (Pierce Rockford, IL) para normalizar y ajustar el contenido de citokina al peso total de cada biopsia. La concentración de cada citoquina en biopsias homogenizadas fue expresada como $\mathrm{mg} / \mathrm{ml}$. El rango de detección fue entre de 20 a $2000 \mathrm{mg} / \mathrm{ml}$. IL12, IL-2, IL-5, IL-10, IFN- $\gamma$ e IL-4, fueron determinados en los sobrenadantes de las biopsias mediante ELISA (BD Biosciences Pharmingen, San Diego, CA) utilizando citoquina humana recombinantes como controles positivos para el desarrollo de curvas estándar, de acuerdo a las instrucciones del fabricante. Los ensayos no poseían reacciones cruzadas detectables con otras citoquinas. La concentración final de citoquinas fue expresada como $\mathrm{pg} / \mathrm{mg}$ de proteína total en cada biopsia.

Test Cutáneos. A todos los pacientes se les realizó un test cutáneo (TC) mediante técnica de prick test establecida, según recomendaciones habituales en relación al tipo de lanceta, técnica de inoculación del alergeno, lectura, registro e informe del examen laboratorios (CBF LATI, España). El estudio fue realizado en el Laboratorio Respiratorio Pediátrico del Hospital Clínico de la PUC por una enfermera entrenada. Se utilizaron 40 alergenos, incluyendo inhalantes domiciliarios, pastos, malezas, árboles, alimentos, látex, histamina como control positivo y solución glicerinada como control negativo (laboratorios CBF LATI, España) que fueron aplicados en la superficie volar del antebrazo. Se midió la reacción a los 15 minutos y se consideró positiva una induración de $3 \mathrm{~mm}$ o más de diámetro (por sobre el control negativo). Se suspendieron medicamentos que podrían interferir con el resultado: antihistamínicos, prednisona, imipramina, tofranil, derivados de clorpromazina, astemizol, esteroides locales, ranitidina y famotidina ${ }^{11}$.

$\boldsymbol{I g} \boldsymbol{E}$. Al momento de la endoscopia a todo paciente se le tomó una muestra de sangre para determinar la IgE total (Ul/ml), mediante ensa- 
yo inmunométrico enzimático quimioluminiscente (Immulite-DPC). Los pacientes con valores de IgE total mayores a puntos de corte por edad fueron considerados IgE positivo.

Cuestionarios clínicos. A los pacientes y/o sus familiares se les efectuó cuestionarios de antecedentes personales y familiares de alergia cutánea, respiratoria, a insectos y a alimentos, ampliamente validados y de uso internacional ${ }^{12}$.

Definición de hipersensibilidad a alimentos (HA). Se evaluó la hipersensibilidad a alimentos mediante los diferentes marcadores y/o instrumentos de alergia antes descritos (TC, IgE, cuestionarios clínicos). Se consideró con HA a pacientes con eosinófilos distinto de 0 más alguna de las siguientes 2 alternativas: TC positivo a alergenos alimentarios, entre 1 y 3 antígenos más la presencia de IgE positiva, o TC positivos a alergenos alimentarios mayor o igual a 4 antígenos. La presencia de eosinófilos en ausencia o presencia de otros marcador, no se consideró suficiente para el diagnóstico de HA.
Análisis estadístico. Para la comparación de variables categóricas se utilizó el test $\chi^{2}$. Para variables continuas no paramétricas se utilizó el test Mann-Whitney. Se consideró significativo el valor de $p<0,05$.

\section{Resultados}

Pacientes. Se incluyeron 97 niños consecutivos, entre 3 a 18 años, con promedio de edad de $11,7 \pm 3$ años, de los cuales 43 (44,3\%) eran hombres. Los pacientes fueron divididos en 2 grupos: pacientes sin HA y pacientes con HA según el criterio establecido previamente. Un $12,4 \%$ de los niños presentó HA. Las características demográficas se resumen en la tabla 1. No hubo diferencias entre ambos grupos.

Hallazgos endoscópicos. Los hallazgos endoscópicos se resumen en la tabla 2. La mayoría de los pacientes presentaron una endoscopia normal $(54,1 \%$ en pacientes $\sin \mathrm{HA}$ vs $58,3 \%$ en pacientes con HA, $\mathrm{p}=\mathrm{NS}$ ) y no

Tabla 1. Características demográficas de los pacientes

\begin{tabular}{llllllr}
\hline Característica & \multicolumn{2}{c}{ HA negativo } & \multicolumn{2}{c}{ HA positivo } & \multicolumn{2}{c}{ Total } \\
& n & (\%) & n & (\%) & n & (\%) \\
\hline$N^{\circ}$ & 85 & $(87,6)$ & 12 & $(12,4)$ & 97 & $(100)$ \\
Edad en años, $x \pm$ DS & $11,8 \pm 3$ & & $11,08 \pm 3,3$ & $11,7 \pm 3$ & \\
Hombres & 38 & $(44,7)$ & 5 & $(41,7)$ & 43 & $(44,3)$ \\
Antecedente familiar de alergia & 79 & $(92,9)$ & 11 & $(91,7)$ & 90 & $(92,8)$ \\
Antecedentes personales de alergiaa & 10 & $(11,8)$ & 4 & $(33,3)^{*}$ & 14 & $(14,4)$ \\
Edad < 10 años & 25 & $(29,4)$ & 4 & $(33,3)$ & 29 & $(29,9)$ \\
\hline
\end{tabular}

asegún cuestionario clínico, * $p<0,05$

Tabla 2. Hallazgos endoscópicos

\begin{tabular}{lcccccc}
\hline Hallazgos endoscópicos & \multicolumn{2}{c}{ HA negativo } & \multicolumn{2}{c}{ HA positivo } & \multicolumn{2}{c}{ Total } \\
& n & (\%) & n & (\%) & n & (\%) \\
\hline$N^{\circ}$ & 85 & $(87,6)$ & 12 & $(12,4)$ & 97 & $(100)$ \\
Normal & 46 & $(54,1)$ & 7 & $(58,3)$ & 53 & $(54,6)$ \\
Nodularidad antral & 15 & $(17,7)$ & 2 & $(16,7)$ & 17 & $(17,5)$ \\
Úlcera gastroduodenal $^{\text {a }}$ & 10 & $(11,8)$ & 1 & $(8,3)$ & 11 & $(11,4)$ \\
Gastropatíab $^{b}$ & 3 & $(3,5)$ & 1 & $(8,3)$ & 4 & $(4,1)$ \\
Duodenopatía $^{c}$ & 4 & $(4,7)$ & 0 & $(0)$ & 4 & $(4,1)$ \\
Otros & 7 & $(8,2)$ & 1 & $(8,3)$ & 8 & $(8,3)$ \\
\hline
\end{tabular}

aactiva o cicatriz, balteraciones inespecíficas mucosa gástrica, calteraciones inespecíficas mucosa duodenal. $p=$ NS 


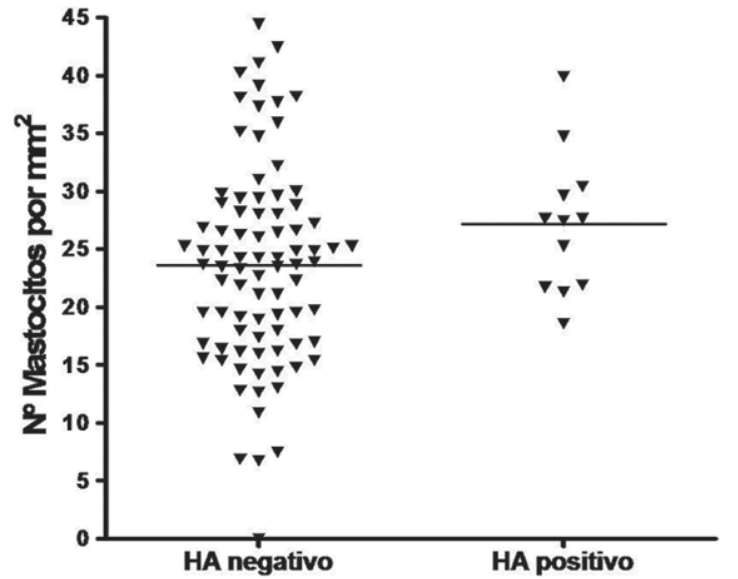

Figura 1. Distribución y media de mastocitos en la mucosa gástrica; número de mastocitos por $\mathrm{mm}^{2} . \mathrm{p}=\mathrm{NS}$.

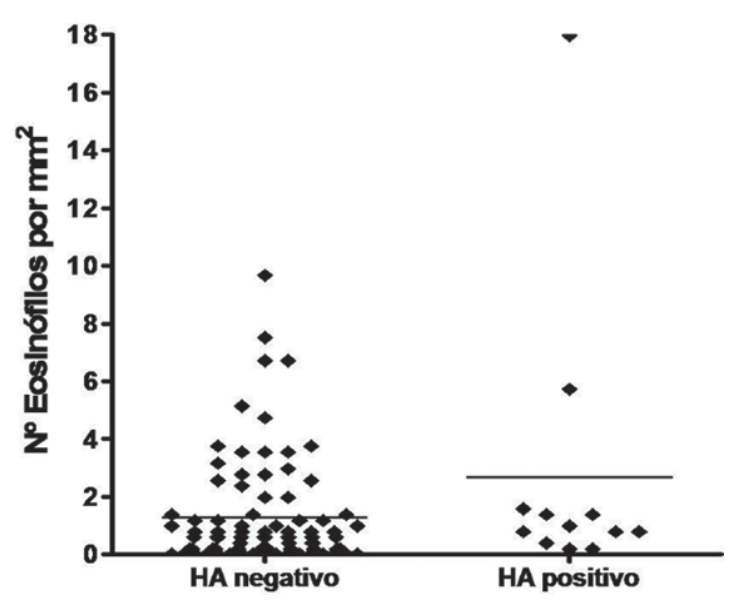

Figura 2. Distribución y media de eosinófilos en la mucosa gástrica; número de eosinófilos por $\mathrm{mm}^{2} . \mathrm{p}=\mathrm{NS}$. hubo diferencias significativas en ningún diagnóstico endoscópico entre ambos grupos.

Hallazgos histológicos. El promedio de mastocitos en la mucosa gástrica fue similar en el grupo con y $\sin$ HA $(27,2 \pm 5$ y $23,6 \pm 8$ mastocitos por $\mathrm{mm}^{2}$ respectivamente; $\mathrm{p}=\mathrm{NS}$ ) (figura 1). Por definición todos los pacientes con HA tenían eosinófilos presentes en la mucosa gástrica antral, pero estos estaban presentes en un $74,1 \%$ de los pacientes sin HA $(\mathrm{p}<0,05)$. Al evaluar la concentración de eosinófilos en la mucosa gástrica, no se observó diferencia significativa entre ambos grupos: $1,28 \pm 1,8$ eosinófilos por $\mathrm{mm}^{2}$ en el grupo sin HA y $2,67 \pm 5$ eosinófilos por $\mathrm{mm}^{2}$ en el grupo con HA; $\mathrm{p}=\mathrm{NS}$ (figura 2). Los hallazgos histológicos se resumen en la tabla 3.

Citoquinas en mucosa gástrica. Como se muestra en la tabla 4, el nivel en mucosa de las citoquinas analizadas fue más baja en el grupo con HA, aunque sólo alcanzó significación estadística para la IL-2 $(451,2 \pm 639,2 \mathrm{pg} / \mathrm{mg}$ en el grupo sin HA vs $193,9 \pm 233,6 \mathrm{pg} / \mathrm{mg}$ en pacientes con HA; $\mathrm{p}<0,05)$.

Cuestionarios clínicos de AA. Para este análisis fueron considerados 2 cuestionarios: el cuestionario de antecedentes de AA personal y el de antecedentes de AA familiar. En el análisis de los cuestionarios de AA personal, un $33,3 \%$ de los niños con HA consideró tener antecedentes personales de AA versus un $11,8 \%$ de los sin HA ( $p<0,05)$. No hubo diferencias en los grupos de HA al evaluar el cuestionario de AA familiar (tabla 1). De los pacientes que se consideran a sí mismos con

Tabla 3. Hallazgos histológicos

\begin{tabular}{|c|c|c|c|c|c|c|}
\hline \multirow[t]{2}{*}{ Hallazgos histológicos } & \multicolumn{2}{|c|}{ HA negativo } & \multicolumn{2}{|c|}{ HA positivo } & \multicolumn{2}{|c|}{ Total } \\
\hline & $\mathbf{n}$ & (\%) & $\mathbf{n}$ & $(\%)$ & $\mathbf{n}$ & (\%) \\
\hline $\mathrm{N}^{\circ}$ & 85 & $(87,6)$ & 12 & $(12,4)$ & 97 & $(100)$ \\
\hline Gastritis crónica & 51 & $(60)$ & 8 & $(66,7)$ & 59 & $(60,8)$ \\
\hline Infiltración polimorfonuclear & 33 & $(38,8)$ & 2 & $(16,7)$ & 35 & $(36,1)$ \\
\hline Infección por H. pylori & 37 & $(43,5)$ & 4 & $(33,3)$ & 41 & $(42,3)$ \\
\hline Metaplasia intestinal & 0 & $(0)$ & 0 & $(0)$ & 0 & $(0)$ \\
\hline Atrofia & 10 & $(11,8)$ & 2 & $(16,7)$ & 12 & $(12,4)$ \\
\hline Presencia eosinófilos & 63 & $(74,1)$ & 12 & $(100)^{*}$ & 75 & $(77,1)$ \\
\hline Concentración eosinófilos ${ }^{a}$ & $1,28 \pm 1,8$ & & $2,67 \pm 5$ & & $=2,5$ & \\
\hline Concentración mastocitos ${ }^{a}$ & $23,6 \pm 8$ & & $27,2 \pm 5$ & & $=8,5$ & \\
\hline
\end{tabular}

a expresados en $n^{\circ} \times m^{2},{ }^{*} p<0,05$ 
Tabla 4. Expresión de citoquinas en mucosa gástrica

\begin{tabular}{lrrl}
\hline $\begin{array}{l}\text { Citoquinas } \\
\overline{\mathbf{x}}+\mathbf{D} \mathbf{E}^{*}\end{array}$ & $\begin{array}{c}\text { HA negativo } \\
\mathbf{n}=\mathbf{8 5}\end{array}$ & $\begin{array}{c}\text { HA positivo } \\
\mathbf{n}=\mathbf{1 2}\end{array}$ & valor $\mathbf{p}$ \\
\hline $\mathrm{IL}-2$ & $451,2 \pm 639,2$ & $193,9 \pm 223,6$ & 0,04 \\
IL-12 & $124,5 \pm 167,4$ & $88,5 \pm 111,9$ & 0,5 \\
IFN- $\gamma$ & $37,3 \pm 45,6$ & $31,4 \pm 26,6$ & 0,9 \\
IL-4 & $29,4 \pm 47,4$ & $15,2 \pm 21,4$ & 0,7 \\
IL-5 & $215,8 \pm 393,6$ & $78,7 \pm 128,6$ & 0,08 \\
IL-10 & $66,8 \pm 79,9$ & $68,9 \pm 56,4$ & 0,4 \\
\hline
\end{tabular}

* pg/mg de proteína total, promedio \pm 1 desviación estándar.

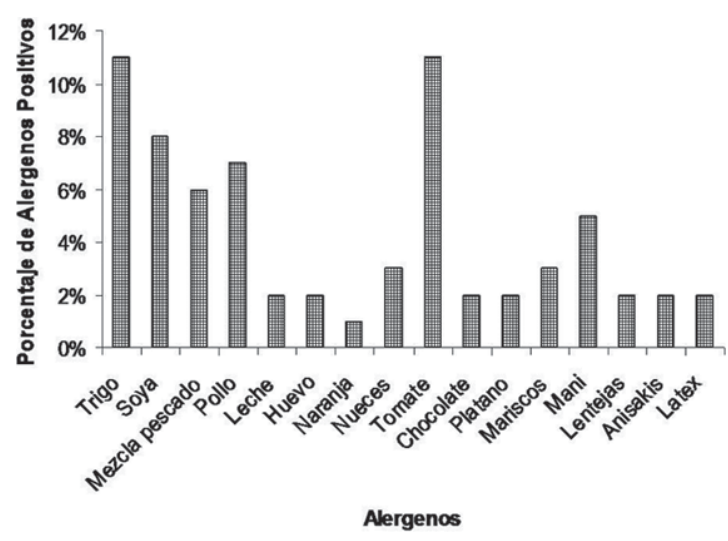

Figura 3. Alergenos alimentarios positivos según resultados de los test cutáneos, expresados en porcentaje.

alergia a alimentos medido por el cuestionario de antecedentes de AA personal, sólo un 28,6\% cumplió criterios de HA.

Test cutáneos. De los 40 alergenos evaluados, en este estudio sólo se analizaron 16 alergenos alimentarios por razones de diseño. Un $38 \%$ de los pacientes tuvo positivo a lo menos 1 alergeno alimentario según el test cutáneo. Los alergenos positivos más prevalentes fueron el trigo $(11,3 \%)$, tomate $(11,3 \%)$, soya $(8,3 \%)$ y pollo $(7,2)$ (figura 3$)$.

\section{Discusión}

En este estudio prospectivo fueron evaluados 97 niños asintomáticos desde el punto de vista de alergia a alimentos y divididos según definición operacional en pacientes con y sin hipersensibilidad a alimentos. Un 12,4\% de la población estudiada cumplió el criterio de HA. Esta cifra es mayor a la encontrada por Bock et $\mathrm{al}^{13}$, donde aproximadamente un $6 \%$ de la población de menores de 3 años presentaría alergia a alimentos con manifestaciones clínicas propias de esta enfermedad. Esto demuestra que no todos los pacientes con HA desarrollan enfermedad clínica y los parámetros evaluados en este estudio podrían constituir un factor de riesgo para desarrollar AA.

En nuestro estudio, no encontramos diferencias en la edad ni sexo, entre ambos grupos. Al evaluar los pacientes en menores versus mayores de 10 años, se observó que no hay diferencia en la prevalencia de $\mathrm{HA}$, sugiriendo una incidencia similar de HA a través del desarro1lo, a diferencia de lo descrito en otros estudios de alergia donde la prevalencia de las alergias y de HA sería mayor en niños menores de 10 años e iría disminuyendo hacia la adultez ${ }^{14,15}$.

Si aceptamos como válido el criterio diagnóstico empleado, debemos concluir que los hallazgos endoscópicos son de escasa utilidad en el diagnóstico de HA. La gran mayoría de los pacientes tuvo endoscopia normal. Esto difiere con lo descrito en otros estudios donde hallazgos inespecíficos como hiperemia y edema de la mucosa gastroduodenal han sido descritos en la mucosa de pacientes con alergia clínica a alimentos o con marcadores de $\mathrm{HA}^{16}$. Los hallazgos endoscópicos no pueden ser utilizados para establecer o descartar el diagnóstico.

Las biopsias endoscópicas están sujetas al clásico error de muestreo ya que representan áreas focales de la mucosa gástrica. En las enfermedades alérgicas del tubo digestivo, la infiltración eosinofílica puede afectar desde el esófago hasta el ano; sin embargo, en pacientes con síntomas digestivos altos y sospecha de $\mathrm{HA}$, el estómago parece estar mayoritariamente afectado (a nivel histológico) aún en ausencia de hallazgos macroscópicos ${ }^{9,14}$. En este estudio la concentración de eosinófilos en la mucosa gástrica fue similar entre ambos grupos. Esto concuerda con resultados observados en mucosa gástrica y colónica en estudios previos ${ }^{15,17}$. Es posible que la activación de estas células en la mucosa sea diferente entre ambos grupos, explicando los diferentes efectos clínicos ${ }^{15}$. La 
alta frecuencia de eosinófilos en la mucosa gástrica de pacientes sin HA $(74,1 \%)$, concuerda con otros estudios realizados sugiriendo que es normal la presencia de eosinófilos en mucosa gastrointestinal sana ${ }^{18-20}$.

La concentración de mastocitos fue similar en la mucosa gástrica de ambos grupos, hallazgo que concuerda con un estudio realizado por Bischoff y cols ${ }^{15}$. Los mastocitos son células que participan en la respuesta de hipersensibilidad inmediata mediada por IgE produciendo una serie de mediadores que llevan a una respuesta inflamatoria ${ }^{1}$. La similitud podría explicarse por la falta de contacto reciente con el alergeno causal en el grupo con HA.

La disminución significativa en la concentración de IL-2 $(\mathrm{p}=0,04)$ en el grupo con HA pudiera sugerir que los niños de este grupo tienden a disminuir el predominio Th1 habitual en niños sanos, aunque la ausencia de diferencia en la concentración de otras citoquinas Th1 (IL-12 e IFN- $\gamma$ ) o Th2 (IL-4 e IL-5), sumado a la gran variabilidad en los resultados individuales obliga a ser cauteloso en la interpretación. El estudio sistemático de la IL-2 a nivel de mucosas nos permitirá en el futuro entender mejor los mecanismos inmunológicos de la alergia a alimentos.

Maciorkowska et al, reportaron concentraciones altas de IL-2 en mucosa gástrica en grupos con infección por $H$. pylori con y sin alergia a alimentos comparados con alergia a alimentos sin infección por H. pylori y un grupo control. Al igual que en el presente estudio no se encontraron diferencias significativas para IL-5 entre los distintos grupos ${ }^{21}$.

De los antecedentes familiares de alergia (cutánea, respiratoria, a alimentos y a insectos) llama la atención su altísima frecuencia en ambos grupos ( $92,8 \%$ de la población estudiada), lo que hace a este dato de escasa utilidad clínica. De los pacientes que se consideraron a sí mismos con alergia a alimentos según el cuestionario de antecedentes de AA personal, sólo un $28,6 \%$ cumplió criterios de HA, aunque la frecuencia clínica de AA fue significativamente mayor en el grupo con HA. Esto demuestra una alta percepción de enfermedad alérgica en la población chilena y la necesidad de ser crítico respecto a este antecedente.
Un 38\% de la población estudiada tuvo reacción cutánea con al menos 1 alergeno alimentario. Los test cutáneos a alimentos tienen un alto valor predictivo negativo ${ }^{22}$, sin embargo, menos del $50 \%$ de los niños con test cutáneos positivos a alimentos se han podido correlacionar con la prueba de doble ciego oral $^{23}$. Esto explicaría la alta prevalencia de TC positivos sin necesariamente reflejar la prevalencia de AA en nuestra población.

En los pacientes estudiados los alergenos positivos más frecuentes fueron el trigo, el tomate, la soya y el pollo. Estos resultados son comparables a los de un estudio realizado en Chile donde los alergenos más frecuente hallados en población pediátrica igual o mayor de 5 años eran la soya, el trigo y la naranja ${ }^{24}$. En otras poblaciones estudiadas los alergenos más prevalentes en USA eran la leche, el huevo y el maní ${ }^{24}$, mientras que en México la leche, el pescado y los mariscos ${ }^{25}$. Esto es importante ya que cada población tiene diferente prevalencia de TC positivos a diferentes alergenos lo que puede ser explicada por los diferentes hábitos alimentarios.

En conclusión, la HA en niños enviados a endoscopia digestiva alta por síntomas digestivos, está presente en un $12,4 \%$. Los niños con HA presentan similares características demográficas, similares hallazgos endoscópicos, similar distribución de eosinófilos y mastocitos en mucosa gástrica y una menor polarización Th1 medida por citoquinas linfocitarias que los niños sin HA. La percepción personal de alergia se correlaciona, en un pequeño grupo de pacientes, con los hallazgos de marcadores de HA. Finalmente, el estudio sistemático de marcadores clínicos de alergia clínica a alimentos e HA permitirá evitar el sobrediagnóstico y las terapias asociada a ello.

\section{Referencias}

1.- Kay $A B$ : Allergy and allergic diseases, first of two parts. N Engl J Med 2001; 344: 30-7.

2.- Asher MI, Montefort S, Bjorsten B, et al: Worldwide time trends in the prevalence of symptoms of asthma, allergic rhinoconjunctivitis, and eczema in childhood: ISAAC Phases One and Three repeat multicountry cross-sectional surveys. Lancet 2006; 368: 733-43. 
3.- No authors listed: Worldwide variation in prevalence of symptoms of asthma, allergic rhinoconjunctivitis, and atopic eczema: ISAAC. The International Study of Asthma and Allergies in Childhood (ISAAC) Steering Committee. Lancet 1998; 351: 1225-32.

4.- Arenillas S, Godoy A, Einisman H, García D, Harris $P$ : Regulación de la respuesta inmune frente a la infección por Helicobacter pylori. Rev Chil Pediatr 2002; 73: $108-15$

5.- Romagnani $S$ : Inmunologic influences on allergy and the Th1/Th2 balance. J Allergy Clin Immunol 2004; 113: 395-400.

6.- Johansson S, Hourihane J, Bousquet J, et al: A revised nomenclature for allergy. Allergy 2001; 56: 813-24.

7.- Sicherer S: Food allergy. Lancet 2002; 360: 701-10.

8.- Sampson HA: Food Allergy. Part 2: diagnosis and management, J Allergy Clin Immunol 1999; 103: 98199.

9.- Bishoff S, Crowe S: Gastrointestinal food allergy: new insights into pathophysiology and clinical perspectives. Gastroenterol 2005; 128: 1089-113.

10.- Walls A, Jones D, Williams J, Church M, Holgate S: Inmunohistochemical identification of mast cells in foremaldehyde-fixed tissue using monoclonal antibodies specific for tryptase. J Pathol 1990; 162: 119-26.

11.- Nelson H: Variables in allergy skin testing. Immunol Allergy Clin North Am 2001; 21: 281-90.

12.- Blaiss M: Approach to the allergic patient. En: Lieberman P, Anderson JA. Allergic Diseases. Diagnosis and treatment. Totowa New Yersey: Editorial Humana Press 1997; 15-26.

13.- Bock $S$ : Prospective appraisal of complaints of adverse reactions to foods in children during the first 3 years of life. Pediatrics 1987; 79: 683-8.

14.- Sampson H: Update on food allergy. J Allergy Clin Immunol 2004; 113: 805-19.

15.- Bischoff S, Mayer J, Wedemeyer J, et al: Colonoscopic allergen provocation (COLAP): a new diagnostic approach for gastrointestinal food allergy. Gut 1997; 40: $745-53$

16.- Corrado G, Luzzi I, Lucarelli S, et al: Positive association between Helicobacter pylori infection and food allergy in children. Scand J Gastroenterol 1998; 33: 1135-9.

17.- Debrosse C, Case J, Putnam P, Collins M, Rothenberg M: Quantity and distribution of eosinophils in the gastrointestinal tract of children. Pediatr Dev Pathol 2006; 9: 210-8.

18.- Kato M, Kephart $G$, Talley $N$, et al: Eosinophil infiltration and degranulation in normal human tissue. Anat Rec 1998; 252: 418-25.

19.- Mishra A, Hogan S, Lee J, Foster P, Rothenberg M: Fundamental signals that regulate eosinophil homing to the gastrointestinal tract. J Clin Invest 1999; 103: 1719-27.

20.- Rothenberg M: Gatrointestinal eosinophils. Allergy 2001; 56: Suppl 67: 21-2.

21.- Maciorkowska E, Panasiuk A, Kaczmarski M: Concentrations of gastric mucosal cytokines in children with food allergy and Helicobacter pylori infection. World J Gastroenterol 2005; 11: 6571-6.

22.- Hill D, Hosking C, Reyes-Benito L: Reducing the need for food allergen challenges in Young children: a comparison of in vitro with in vivo tests. Clin Exp Allergy 2001; 31: 1031-5.

23.- Bock S, Lee W, Remigio L, Holst A, May C: Appraisal of skin test with food extract for diagnosis of food hypersensitivity. Clin Allergy 1978; 8: 559-64.

24.- Martínez J, Méndez C, Talesnik E, Campos E, Viviani $P$, Sánchez I: Pruebas cutáneas de hipersensibilidad inmediata en una población pediátrica seleccionada. Rev Méd Chile; 2005; 133: 195-201.

25.- Ávila L, Pérez J, Del Río B, Rosas M, Lerma L, Sierra $J$ : Hypersensitivity detected by skin tests to food in allergic patients in the Hospital Infantil de México Federico Gómez. Rev Alerg Mex 2002; 49: 74-9. 